\title{
CT-diagnosed severe skull base bone destruction predicts distant bone metastasis in early $\mathrm{N}$-stage nasopharyngeal carcinoma
}

This article was published in the following Dove Press journal:

OncoTargets and Therapy

I4 November 2016

Number of times this article has been viewed

\author{
Wei Yi, I,* Zhi-Gang Liu, 2,3,* \\ Xian Li, ${ }^{4}$ Jiao Tang, ${ }^{2,3}$ Chang- \\ Bin Jiang,' Jing-Ye $\mathrm{Hu},{ }^{5}$ \\ Zi-Wei Tu, ${ }^{5}$ Hui Wang, ${ }^{2,3}$ \\ Dao-Li Niu,' Yun-Fei Xia ${ }^{5}$ \\ 'Department of Radiotherapy, The \\ First Affiliated Hospital of Guangzhou \\ Medical University, Guangzhou, \\ ${ }^{2}$ Department of Radiotherapy, Hunan \\ Cancer Hospital, The Affiliated \\ Cancer Hospital of Xiangya School of \\ Medicine, Central South University, \\ Changsha, ${ }^{3}$ Key Laboratory of \\ Translational Radiation Oncology, \\ Hunan Province, ${ }^{4}$ Department of \\ Radiology, The First Affiliated Hospital \\ of Guangzhou Medical University, \\ ${ }^{5}$ State Key Laboratory of Oncology \\ in Southern China, Department of \\ Radiotherapy, Sun Yat-Sen University \\ Cancer Center, Guangzhou, People's \\ Republic of China \\ *These authors contributed equally \\ to this work
}

Correspondence: Yun-Fei Xia State Key Laboratory of Oncology in Southern China, Department of Radiotherapy, Sun Yat-Sen University Cancer Center, 65I Dongfeng East Road, Guangzhou 5I0060, People's Republic of China

Tel +862087343336

Email xiayf@sysucc.org.cn

Dao-Li Niu

Department of Radiotherapy, The First Affiliated Hospital of Guangzhou Medical University, I5I Yanjiang Road,

Guangzhou 510120, Guangdong, People's

Republic of China

Tel +86203429 4221

Email daoli_niu@I63.com

\begin{abstract}
Bone metastasis is the most frequent type of distant metastasis in nasopharyngeal carcinoma (NPC). In this study, we investigated the correlation between the skull base bone destruction and the distant bone metastasis in patients with NPC. A total of 449 cases with NPC who were diagnosed and had definitive radiotherapy from 2001 to 2006 were enrolled in this study. The skull base bone destruction was diagnosed by computed tomography (CT) in all cases, and 191 patients also underwent magnetic resonance imaging scan. Kaplan-Meier method was adopted to perform the univariate analysis; Cox regression model was used to perform multivariate analysis to determine whether the skull base bone destruction when diagnosed by CT was an independent impact factor of the distant bone metastases. The group with skull base bone destruction had a distant bone metastases rate of $9.0 \%(14 / 155)$, whereas the group without skull base bone destruction had rate of $4.1 \%$ (12/294). The multivariate analysis showed that the skull base bone destruction, when diagnosed by CT, was an independent impact factor of the distant bone metastases-free survival in the early N-staging cases, but was not an independent impact factor when diagnosed by MRI. The skull base bone destruction diagnosed by CT in patients with NPC had predictive value for the distant bone metastases, especially for the early N-staging cases.
\end{abstract}

Keywords: bone metastasis, skull base bone destruction, radiotherapy, nasopharyngeal carcinoma

\section{Introduction}

The incidence rate of NPC was found to be high in the people of Southern China. ${ }^{1}$ In these regions, the pathological type was poorly differentiated and was characterized by invasive growth and distant metastases. Currently, the treatment for NPC is a comprehensive one that is based on radiotherapy, and there has been an increase in the 5-year overall survival rate from $40 \%$ (in $1960 \mathrm{~s}-1970$ s) to more than $80 \%$ (in 2000s). ${ }^{2}$ According to the clinical and biological behavior of NPC patients following chemoradiotherapy, NPC was subdivided into four types: type I (no primary and regional recurrence and no distant metastasis), type II (primary or regional recurrence and no distant metastasis), type III (no primary and regional recurrence and distant metastasis), and type IV (primary or regional recurrence and distant metastasis). ${ }^{3}$ In the intensity-modulated radiation therapy era, distant metastasis was observed as treatment failure in NPC, and the most frequent type was bone metastasis. ${ }^{4}$ Some scholars have suggested subdividing the M1 stage of NPC and providing a more individualized therapy. ${ }^{5,6}$ Accordingly, the identification of prognostic factors that are more accurately correlated with bone metastasis could help in determining the NPC patients who may benefit from more aggressive chemotherapy or other treatment intervention. 
Computed tomography (CT) and magnetic resonance imaging (MRI) are the important techniques used for establishing diagnosis and predicting prognosis in NPC patients. The CT-evident skull base erosion has been proved to be a significant independent prognostic factor for the regional control and distant metastasis in NPC. ${ }^{7}$ The incidence rate of skull base bone destruction was found to be in the range of $30 \%-40 \%$ on the CT $\operatorname{scan}^{8-10}$ and $55 \%-66 \%$ on the MRI scan. ${ }^{11-13}$ Although intensity-modulated radiotherapy could improve the local control rates, distant metastasis mainly accounts for the failure of NPC treatment. ${ }^{4,14}$ Previous studies demonstrated that bone metastasis is the most frequent distant metastasis noted in NPC (observed in more than $29 \%$ of the cases). ${ }^{4,15}$ So determining the predictors of bone metastasis and giving early intervention is an important research direction. According to the classic theory of "seed and soil", ${ }^{16}$ tumors tend to migrate to organs with suitable microenvironment. The skull base bone has a microenvironment similar to that of the distal bone. We conducted this study to determine the correlation between the skull base bone destruction and distant bone metastasis in NPC patients.

\section{Methods}

\section{Study population}

A total of 449 cases pathologically diagnosed with primary NPC (I-IVa stages) in the First Affiliated Hospital of Guangzhou Medical University and Sun Yat-Sen University Cancer Center from November 2001 to November 2006 were selected. The ethical approval for this study was obtained from the ethics committee of the First Affiliated Hospital of Guangzhou Medical University and the Sun Yat-Sen University Cancer Center. The ethics committee of the First Affiliated Hospital of Guangzhou Medical University and the Sun Yat-Sen University Cancer Center did not require written informed consent be obtained from all patients, as this was a retrospective study, and all data was anonymous. These patients received threedimensional conformal radiotherapy or intensity-modulated radical radiotherapy, with complete follow-up data.

\section{Radiotherapy and chemotherapy}

All patients had been treated by definitive radiation therapy. The nasopharyngeal primary lesions and cervical lymph nodes were treated with three-dimensional conformal radiotherapy. The irradiation was first applied of bilateral face neck joint portals and anterior neck portal, a part plus anterior nose portal, at the dose of 34-36 Gy, then bilateral small face and neck portals avoiding spinal cord and bilateral posterior cervical triangle plus anterior neck portal at the dose of $50 \mathrm{~Gy}$, next the nasopharynx were treated with preauricular portal at the radical cure dose of $70 \mathrm{~Gy}$. An irradiation of basis cranii portal at a dose of 6-8 Gy was done for those patients with obvious skull base bone destruction. For those patients having cervical lymph node metastases, the irradiation dose was increased to 60-70 Gy depending on the size and degree of tumor regression.

Chemotherapy was given depending on the tumor staging. The indication for the chemotherapy was: 1 ) advanced clinical stage (stage III-IV); 2) stage T1-2N1 patients who also received concomitant chemotherapy; 3 ) patients who can tolerate chemotherapy. The regimen of neoadjuvant chemotherapy includes 1-2 cycles of cisplatin $100 \mathrm{mg} / \mathrm{m}^{2}$ day (d) $1+5$-Fu $1 \mathrm{~g} / \mathrm{m}^{2} \mathrm{~d} 1$-d5 every 3 weeks (q3w) (PF) or docetaxol $75 \mathrm{mg} / \mathrm{m}^{2}$ $\mathrm{d} 1+$ cisplatin $75 \mathrm{mg} / \mathrm{m}^{2} \mathrm{~d} 1+5-\mathrm{Fu} 1 \mathrm{~g} / \mathrm{m}^{2} \mathrm{~d} 2-\mathrm{d} 5 \mathrm{q} 3 \mathrm{w}$ (TPF), and the regimen of concomitant chemotherapy includes 2-3 cycles of PF or 5-6 cycles of cisplatin $30 \mathrm{mg} / \mathrm{m}^{2} \mathrm{~d} 1$ every week (DDP). The regimen of adjuvant chemotherapy includes 2-4 cycles of PF or docetaxol $75 \mathrm{mg} / \mathrm{m}^{2} \mathrm{~d} 1+$ cisplatin $75 \mathrm{mg} / \mathrm{m}^{2} \mathrm{~d} 1 \mathrm{q} 3 \mathrm{w}$ (TP). Of the 449 cases, 197 adopted one of the previously mentioned regimens of chemotherapy.

\section{$\mathrm{CT}$ and $\mathrm{MRI}$ evaluation before treatment}

The CT image of skull base erosion was classified into three types: A, bone destruction, CT scans show the bone erosion ranging from intensive bone destruction, through varying degrees of intracranial invasion with $\mathrm{CN}$ involvement, to subtle bone defects without $\mathrm{CN}$ involvement; $\mathrm{B}$, bone sclerosis, $\mathrm{CT}$ scans show the density in the invaded region of the skull base is higher than that of the normal area; and $\mathrm{C}$, both conditions (bone defect and abnormal high density area) occur simultaneously., ${ }^{9,17}$ CT diagnosis at the base of the skull base was performed in all patients from the level of anterior clinoids down to $\mathrm{C} 3$ vertebra at $2.5 \mathrm{~mm}$ intervals parallel to the orbitomeatal line. All cases underwent $\mathrm{CT}$ examination before radiotherapy, and the skull base bone destruction was diagnosed according to the CT results by two or more highly qualified radiologists.

A total of 191 cases also had an MRI inspection at the same time, and bone destruction in these patients was diagnosed based on the corresponding images. Bone invasion was diagnosed based on the following observations on MRI: 1) a defect in the low signal intensity of the cortex on T1W1,2) replacement of high-signal-intensity marrow with low-signal-intensity tissue on $\mathrm{T} 1 \mathrm{~W} 1$, and 3) the abnormal tissue mentioned in (1) and (2) showed moderate contrast enhancement on postcontrast T1W1.

\section{Follow-up and statistical methods}

Follow-up started from the beginning date of the radiotherapy. The median duration of follow-up was 68.5 months (2-102 months). SPSS16.0 (SPSS Inc., Chicago, IL, USA) 
statistical software package was used for the statistical analysis, and Pearson's chi-square test was used for the differential counting. Survival rate was calculated with Kaplan-Meier method, and comparison between the groups was done with log-rank test. The multivariate analysis was performed using Cox regression model and backward likelihood ratio test. All the aforementioned tests were bilateral, and $P<0.05$ was considered to be statistically significant.

\section{Results \\ Case characteristics}

The clinical-pathological characteristics of 449 cases enrolled in this study are shown in Table 1; of which, 155 cases had skull base bone destruction and 294 cases did not. Of the 449 enrolled cases, 55 cases (12.2\%) developed some kind of distance metastasis. Of the 55 cases of distance metastasis, a total of 26 cases had distance bone metastasis. Among 26 cases of distance bone metastasis, 14 cases (9.0\%) had skull base bone destruction and 12 cases (4.1\%) did not.

Table I Relationship between the clinical-pathological features and the skull base bone destruction in 449 cases with NPC

\begin{tabular}{|c|c|c|c|}
\hline \multirow[t]{2}{*}{ Clinical features } & \multicolumn{2}{|c|}{$\begin{array}{l}\text { Skull base bone } \\
\text { destruction }\end{array}$} & \multirow[t]{2}{*}{$P$-value } \\
\hline & Without & With & \\
\hline Age, years & & & 0.843 \\
\hline$<46$ & 142 & 77 & \\
\hline$\geq 46$ & 152 & 78 & \\
\hline Sex & & & 0.239 \\
\hline Male & 208 & 101 & \\
\hline Female & 86 & 54 & \\
\hline Pathology & & & 0.725 \\
\hline Undifferentiated NKC & 267 & 143 & \\
\hline Differentiated NKC & 27 & 12 & \\
\hline Clinical stages & & & $<0.001$ \\
\hline I & 23 & 0 & \\
\hline II & 113 & 0 & \\
\hline III & 118 & 54 & \\
\hline IV & 40 & 101 & \\
\hline T-staging & & & $<0.001$ \\
\hline $\mathrm{TI}$ & 57 & 0 & \\
\hline $\mathrm{T} 2$ & 149 & 0 & \\
\hline $\mathrm{T} 3$ & 74 & 58 & \\
\hline $\mathrm{T} 4$ & 14 & 97 & \\
\hline$N$-staging & & & 0.340 \\
\hline No & 91 & 51 & \\
\hline $\mathrm{NI}$ & 105 & 53 & \\
\hline N2 & 72 & 44 & \\
\hline N3 & 26 & 7 & \\
\hline Chemotherapy & & & $<0.001$ \\
\hline No & 197 & 55 & \\
\hline Yes & 97 & 100 & \\
\hline Distant bone metastases & & & 0.036 \\
\hline No & 280 & 143 & \\
\hline Yes & 14 & 12 & \\
\hline
\end{tabular}

Abbreviations: NKC, nonkeratinizing carcinoma; NPC, nasopharyngeal carcinoma.
There were significant differences between the group with skull base bone destruction and the group without skull base bone destruction.

\section{Influence of skull base bone destruction diagnosed by CT on prognosis}

First, we analyzed the influence of skull base bone destruction diagnosed by CT on the prognostic indicators. The results showed that it had significant influences on the overall survival but not on local relapse-free survival (Figure 1A and B). In distant metastases, the skull base bone destruction only had significant influence on the bone metastases-free survival (Figure 1C and D).

\section{Multivariate analysis of the bone metastasis-free survival}

Cox model had been adopted in the multiple regression analysis to verify whether the skull base bone destruction diagnosed by CT was an independent impact factor of the distant bone metastases, to further eliminate the effects of other factors. The T-staging, N-staging, chemotherapy, and skull base bone destruction were included in the model. The results showed that the skull base bone destruction was an independent impact factor. In addition, N-staging also had significant influence on bone metastases (Table 2).

\section{Influence of skull base bone destruction diagnosed by CT on bone metastasis in different $\mathrm{N}$-staging groups}

It is widely acknowledged that N-staging is a factor that influences the distant metastasis, so we further analyzed the influence of skull base bone destruction diagnosed by CT on bone metastasis in cases of different N-staging. For the cases at early $\mathrm{N}$-staging $\left(\mathrm{N}_{0-1}\right)$, the incidence of bone metastasis was $8.7 \%(9 / 104)$ for the cases with skull base bone destruction, but $2.6 \%(5 / 196)$ for those without destruction, indicating the destruction had great influence on the distant bone metastases-free survival (Figure 2A). But for the cases at advanced $\mathrm{N}$-staging $\left(\mathrm{N}_{2-3}\right)$, the incidence of bone metastasis was $9.1 \%(5 / 58)$ for the cases with skull base bone destruction, but 7.1\% (7/98) for those without destruction, indicating there was no statistical significance between the groups (Figure 2B).

\section{Influence of skull base bone destruction diagnosed by MRI on prognosis}

Among the total 449 cases, 191 cases also received MRI inspection before radiotherapy, and the skull base bone 
A

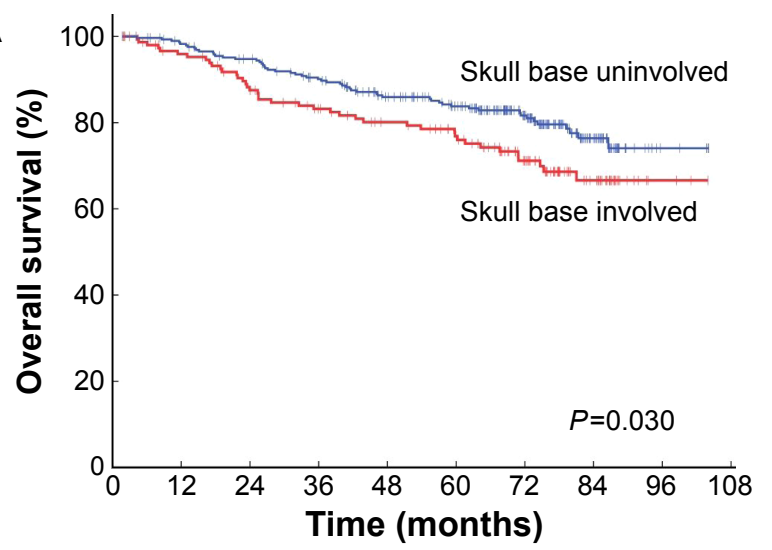

C

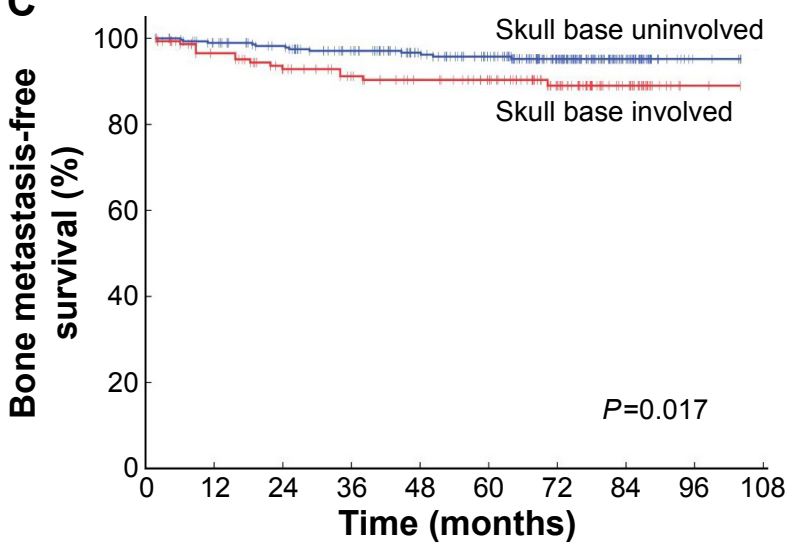

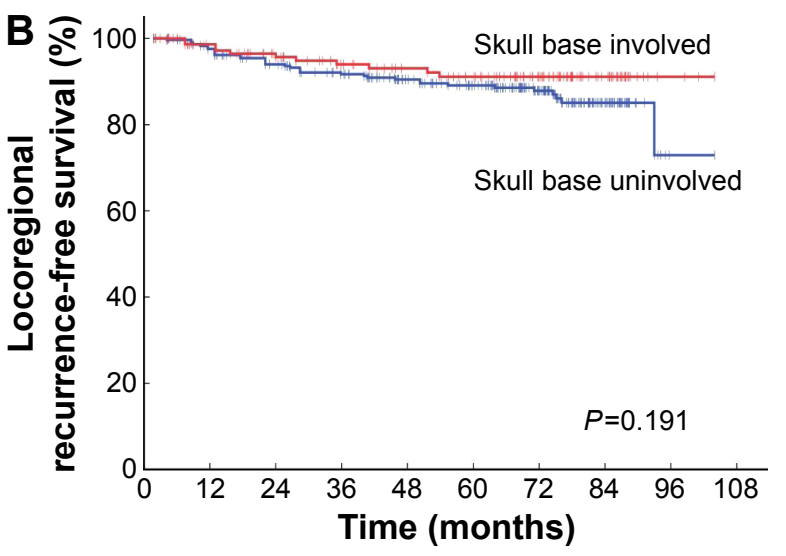

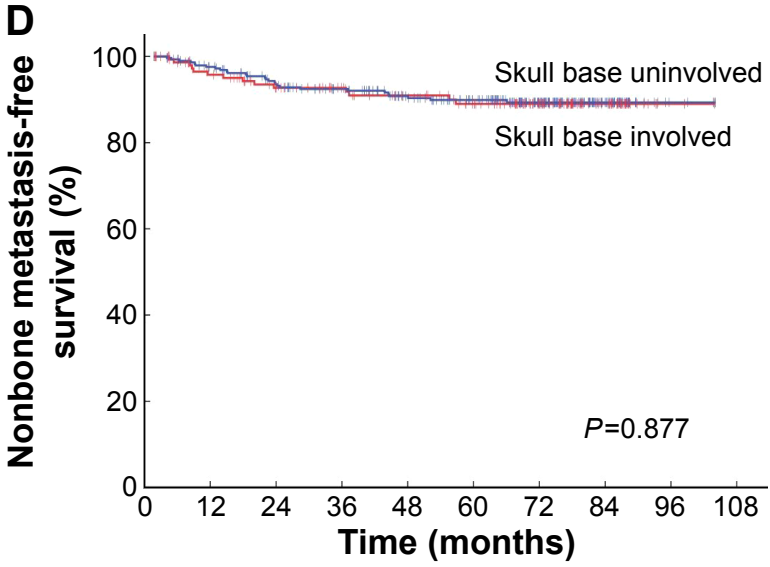

Figure I Influence of skull base bone destruction diagnosed by CT on the distant metastases survival.

Notes: (A) overall survival; (B) local relapse-free survival; (C) bone metastasis-free survival; and (D) nonbone metastasis-free survival.

Abbreviation: $\mathrm{CT}$, computer tomography.

destruction had been diagnosed based on MRI and CT results. Of the 191 cases, 76 were diagnosed with skull base bone destruction according to MRI criteria. Forty-one (21\%) cases had inconsistent diagnoses, mostly showing positive bone metastases on MRI but negative results on CT (37/41) (Figure 3). Among the 191 cases, we analyzed the influences of skull base bone destruction diagnosed by MRI on the local relapse-free survival, the distant metastases-free survival, and the bone metastases-free survival, and the results did not show significant influences (Figure 4A-C). However, the destruction diagnosed by CT still showed obvious influence on the distant bone metastases-free survival (Figure 4D).

Table 2 Multivariate analysis of the factors influencing distant bone metastases

\begin{tabular}{lll}
\hline Clinical factors & \multicolumn{2}{l}{ Distant bone metastases } \\
\cline { 2 - 3 } & RR (95\% Cl) & P-value \\
\hline T-staging & $0.978(0.530-1.806)$ & 0.944 \\
N-staging & $1.436(0.950-2.173)$ & 0.086 \\
Chemotherapy & $0.936(0.404-2.167)$ & 0.878 \\
Skull base bone destruction & $2.478(1.146-5.358)$ & 0.021 \\
\hline
\end{tabular}

Abbreviations: $\mathrm{RR}$, relative risk; $\mathrm{Cl}$, confidence interval.

\section{Discussion}

The skull base bone destruction is an important predictor of the prognosis of NPC, occupying an important position in the Union for International Cancer Control and China staging. Its appearance marked the presence of a local tumor at stage T3, a significantly worse prognosis and increasing possibility of local recurrence and distant metastases. ${ }^{7}$ But there are relatively few studies with regard to the relationship between the skull base bone destruction and the distant bone metastases. In this study, we found that there is a clear correlation between the two conditions, ie, the patients with local skull base bone destruction, diagnosed by CT, had a significantly increased risk of distant bone metastases, whose influence was even evident than $\mathrm{N}$-staging. However, the skull base bone destruction did not influence the risk of nonbone distance metastasis significantly. The mechanism of this phenomenon may be for the following reasons: first, from an anatomical point of view, the skull base bone is rich in bone marrow and blood, one of the origins of distant metastases of NPC; second, the cases with skull base bone destruction, especially at the early N-stage of NPC, might have certain "osseous addiction". The biological 

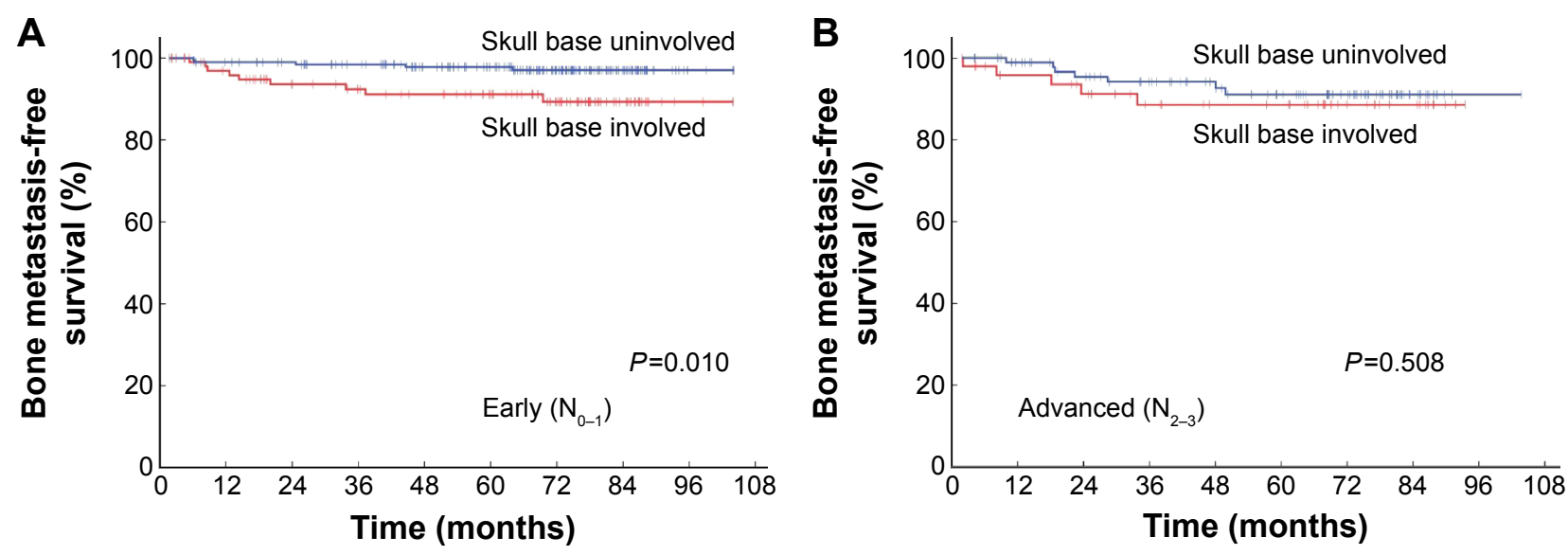

Figure 2 Influence of skull base bone destruction diagnosed by CT on the bone metastases-free survival according to early $N$-stages $\left(\mathrm{N}_{0-1}\right)(\mathbf{A})$ and advanced $\mathrm{N}$-stages $\left(\mathrm{N}_{2-3}\right)(\mathbf{B})$. Abbreviation: $\mathrm{CT}$, computer tomography.

characteristics enable the destruction of the normal bone structure, favoring the building of a suitable microenvironment in the bone marrow. According to the "seed and soil" theory proposed by Paget, ${ }^{16}$ the distant metastases of such "seed" are also more likely to be present in the bone after entering into the blood circulation.

Currently, the imaging methods used for the diagnosis of skull base bone destruction are mainly CT and MRI scans. MRI is more sensitive in detecting the invasion of early tumor to bone marrow, and has a detection rate higher than $\mathrm{CT}$ at $50 \%-70 \% .^{11,13}$ MRI can also identify slight destruction, thus decreasing the prognosis value. Therefore skull base bone destruction could be an independent prognostic factor only when the severe ones were sorted. ${ }^{12}$ In this study, a total of 191 cases underwent CT and MRI at the same time. After comparing the diagnoses, we found that 41 cases $(21 \%)$ showed inconsistent results, mostly with positive bone metastases for MRI but negative for CT. In the analysis of the bone metastases-free survival, there was no statistical difference between the groups with and without skull bone destruction when diagnosed by MRI, but significant difference was observed between the groups when diagnosed by CT (Figure 4D). For the group with inconsistent diagnoses by $\mathrm{CT}$ and MRI, the bone metastases-free survival curve was close to that of the group without skull base bone destruction diagnosed by MRI, but significantly different from that of the group with skull base bone destruction diagnosed by $\mathrm{CT}$. This may be due to high sensitivity of the MRI method in diagnosing skull base bone destruction, and its ability to detect very slight bone destruction. Because the nasopharyngeal

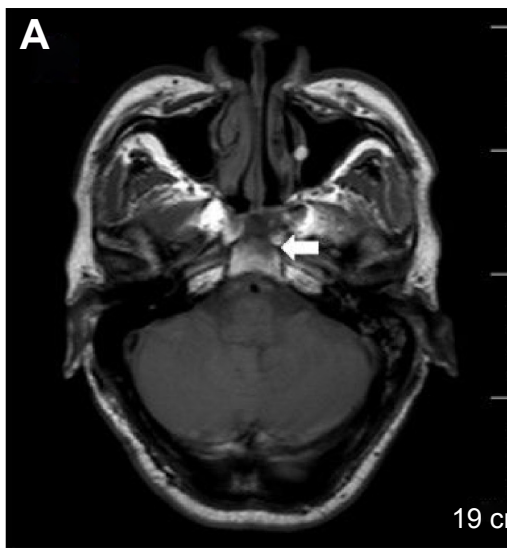

MR T1WI

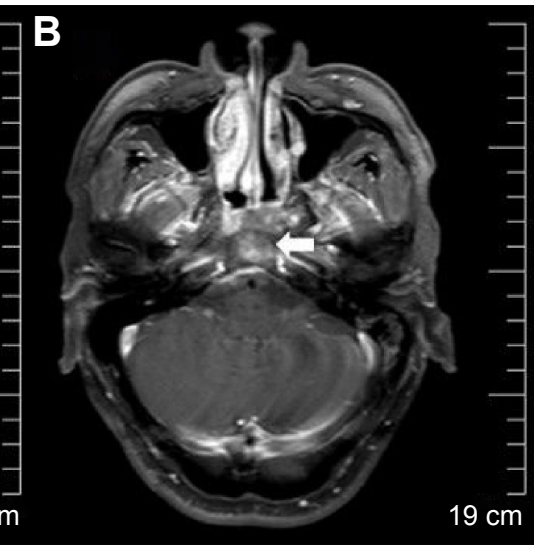

MR T1WI + CE Fat saturation sequence

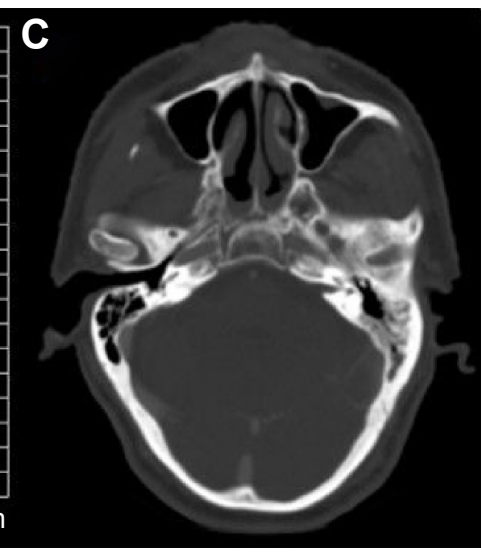

CT imagine

Figure 3 A typical case showing inconsistent diagnosis of skull base bone destruction by CT and MR imaging.

Notes: (A) TI-weighted MR image shows replacement of high-signal-intensity marrow with low-signal-intensity tissue in clivus (white arrow); (B) Contrast-enhanced fatsaturated TI-weighted MR image shows moderate contrast enhancement in clivus (white arrow), which indicates bone invasion; (C) CT image shows negative result for bone destruction or sclerosis.

Abbreviations: $\mathrm{CE}$, contrast enhanced; CT, computer tomography; MR, magnetic resonance. 
A

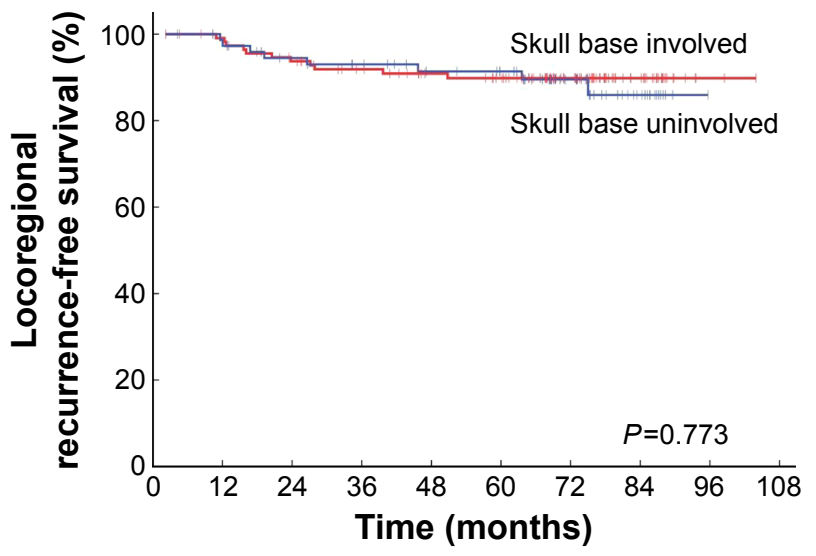

C

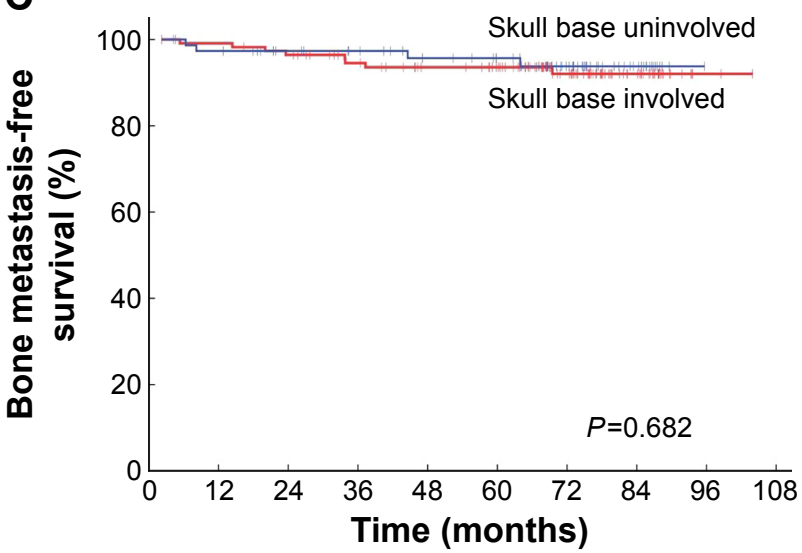

B

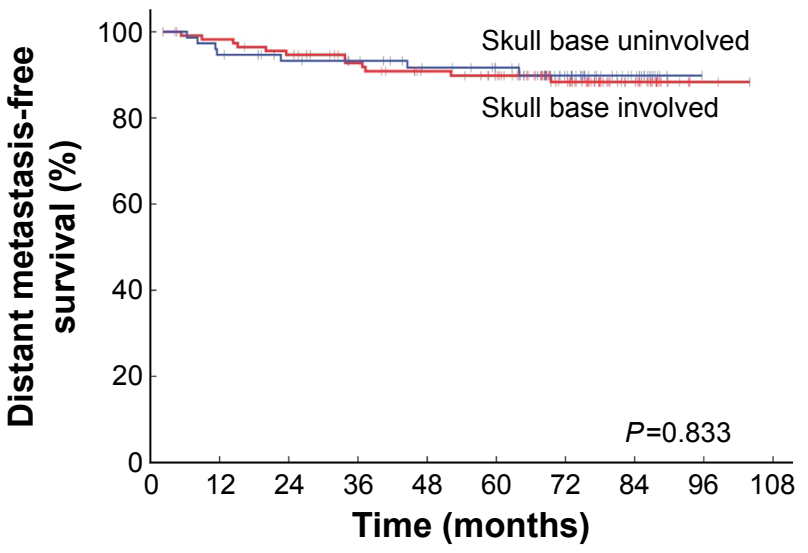

D

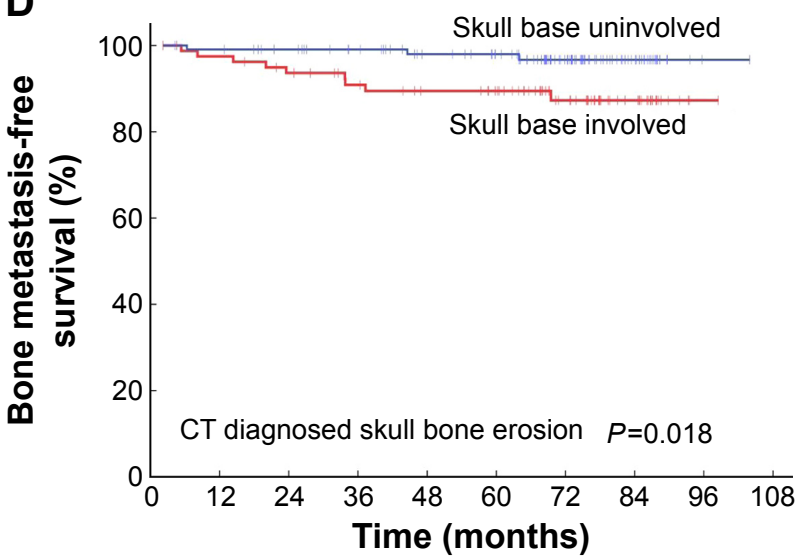

Figure 4 Influences of skull base bone destruction in I9I cases diagnosed by CT or MR imaging on the bone metastases-free survival. Notes: (A) The comparison of the local recurrence-free survival, (B) the distant metastases-free survival, (C) the bone metastases-free survival, when skull base bone destruction was diagnosed by MR, and (D) the comparison of the bone metastases-free survival, when skull base bone destruction was diagnosed by CT.

Abbreviations: $\mathrm{CT}$, computer tomography; MR, magnetic resonance.

tumor is located at a distance very close from the base of the skull, slight bone destruction can also be detected early by MRI examination. Such early invasion or destruction was not a reliable marker indicating the "osseous addiction" of the tumor, or may not be enough to cause tumor to spread into the blood circulation from that region. In addition, those cases with skull base bone destruction diagnosed by MRI, had a significantly higher proportion of chemotherapy than cases without destruction as diagnosed by MRI and CT (62.2\% vs $36.5 \%, P=0.015)$. The proportion of applying chemotherapy might have certain influence on the distant bone metastases. ${ }^{4}$

As an impact factor of the distant bone metastasis, the skull base bone destruction has important clinical application value in nasopharyngeal carcinoma. ${ }^{17}$ First, there are reliable methods for the prevention and treatment of distant bone metastasis. A study by Gnant et al on breast cancer suggested that bisphosphonates could be effective for the prevention of bone related events (radiation to bone, spinal cord compression, fracture, and surgery to bone).$^{18}$ The studies on nasopharyngeal carcinoma also suggested that bisphosphonates could effectively reduce the tumor invasion and metastasis ability, ${ }^{19}$ and improve the overall survival, the disease-free survival, as well as reduce bone events in patients with bone metastases. ${ }^{20}$ The prophylactic use of bisphosphonates in cases with skull base bone destruction may promote the skull bone repair and also reduce the incidence of bone metastases, thus improving the survival rate. The application of bisphosphonates might be feasible in simultaneously improving both the survival rate and survival quality by reducing or replacing adjuvant chemotherapy. Recently, some new biomarkers like osteopontin were found to be a useful predictor for bone metastasis in nasopharyngeal carcinoma. ${ }^{21}$ Their combination with skull base bone destruction may predict bone metastasis more accurately. Though, further prospective studies will have to be carried out to confirm it. 


\section{Conclusion}

The results of the present retrospective analysis indicate that the patients with local skull base bone destruction diagnosed by $\mathrm{CT}$ had a significantly increased risk of distant bone metastases in early N-staging nasopharyngeal carcinoma. However, as this is a retrospective study, further prospective trials with larger sample size that take into consideration more factors like aggressive chemotherapy and bisphosphonates are warranted.

\section{Acknowledgments}

This work was partially supported by Hunan Administration of Foreign Experts Affairs (number CG144300009) and Guangdong Natural Science Foundation (number 2015A030310473).

\section{Disclosure}

The authors report no conflicts of interest in this work.

\section{References}

1. Wei KR, Zheng RS, Zhang SW, Liang ZH, Ou ZX, Chen WQ. Nasopharyngeal carcinoma incidence and mortality in China in 2010. Chin J Cancer. 2014;33(8):381-387.

2. Su SF, Han F, Zhao C, et al. Treatment outcomes for different subgroups of nasopharyngeal carcinoma patients treated with intensity-modulated radiation therapy. Chin J Cancer. 2011;30(8):565-573.

3. Li ZQ, Xia YF, Liu Q, et al. Radiotherapy-related typing in 842 patients in canton with nasopharyngeal carcinoma. Int $J$ Radiat Oncol Biol Phys. 2006;66(4):1011-1016.

4. Li AC, Xiao WW, Shen GZ, et al. Distant metastasis risk and patterns of nasopharyngeal carcinoma in the era of IMRT: long-term results and benefits of chemotherapy. Oncotarget. 2015;6(27):24511-24521.

5. Shen L, Dong J, Li S, et al. M1 stage subdivision and treatment outcome of patients with bone-only metastasis of nasopharyngeal carcinoma. Oncologist. 2015;20(3):291-298.

6. Pan CC, Lu J, Yu JR, et al. Challenges in the modification of the M1 stage of the TNM staging system for nasopharyngeal carcinoma: a study of 1027 cases and review of the literature. Exp Ther Med. 2012;4(2): 334-338.
7. Teo P, Yu P, Lee WY, et al. Significant prognosticators after primary radiotherapy in 903 nondisseminated nasopharyngeal carcinoma evaluated by computer tomography. Int J Radiat Oncol Biol Phys. 1996;36(2): 291-304.

8. Cheung YK, Sham J, Cheung YL, Chan FL. Evaluation of skull base erosion in nasopharyngeal carcinoma: comparison of plain radiography and computed tomography. Oncology. 1994;51(1):42-46.

9. Altun M, Tenekeci N, Kaytan E, Meral R. Locally advanced nasopharyngeal carcinoma: computed tomography findings, clinical evaluation, and treatment outcome. Int J Radiat Oncol Biol Phys. 2000;47(2): 401-404.

10. Sham JS, Cheung YK, Choy D, Chan FL, Leong L. Nasopharyngeal carcinoma: CT evaluation of patterns of tumor spread. AJNR Am J Neuroradiol. 1991;12(2):265-270.

11. Chong VF, Fan YF. Skull base erosion in nasopharyngeal carcinoma: detection by CT and MRI. Clin Radiol. 1996;51(9):625-631.

12. Li YZ, Cai PQ, Xie CM, et al. Nasopharyngeal cancer: impact of skull base invasion on patients prognosis and its potential implications on TNM staging. Eur J Radiol. 2013;82(3):e107-e111.

13. Chen L, Liu LZ, Mao YP, et al. Grading of MRI-detected skull-base invasion in nasopharyngeal carcinoma and its prognostic value. Head Neck. 2011;33(9):1309-1314.

14. Kam MK, Teo PM, Chau RM, et al. Treatment of nasopharyngeal carcinoma with intensity-modulated radiotherapy: the Hong Kong experience. Int J Radiat Oncol Biol Phys. 2004;60(5):1440-1450.

15. Peng X, Chen S, Du C, et al. Clinical features and prognostic factors in patients with nasopharyngeal carcinoma relapse after primary treatment. Head Neck Oncol. 2013;5(2):21.

16. Paget $\mathrm{S}$. The distribution of secondary growths in cancer of the breast. 1889. Cancer Metastasis Rev. 1989;8(2):98-101.

17. Sham JS, Cheung YK, Choy D, Chan FL, Leong L. Cranial nerve involvement and base of the skull erosion in nasopharyngeal carcinoma. Cancer. 1991;68(2):422-426.

18. Gnant M, Dubsky P, Hadji P. Bisphosphonates: prevention of bone metastases in breast cancer. Recent Results Cancer Res. 2012;192: 65-91.

19. Li XY, Lin YC, Huang WL, et al. Zoledronic acid inhibits proliferation and impairs migration and invasion through downregulating VEGF and MMPs expression in human nasopharyngeal carcinoma cells. Med Oncol. 2012;29(2):714-720.

20. Jin Y, An X, Cai YC, et al. Zoledronic acid combined with chemotherapy bring survival benefits to patients with bone metastases from nasopharyngeal carcinoma. J Cancer Res Clin Oncol. 2011;137(10): 1545-1551.

21. Hou X, Wu X, Huang P, et al. Osteopontin is a useful predictor of bone metastasis and survival in patients with locally advanced nasopharyngeal carcinoma. Int J Cancer. 2015;137(7):1672-1678.
OncoTargets and Therapy

\section{Publish your work in this journal}

OncoTargets and Therapy is an international, peer-reviewed, open access journal focusing on the pathological basis of all cancers, potential targets for therapy and treatment protocols employed to improve the management of cancer patients. The journal also focuses on the impact of management programs and new therapeutic agents and protocols on

\section{Dovepress}

patient perspectives such as quality of life, adherence and satisfaction. The manuscript management system is completely online and includes a very quick and fair peer-review system, which is all easy to use. Visit http://www.dovepress.com/testimonials.php to read real quotes from published authors. 